\title{
Incidence and Risk Factors of Venous Thromboembolism in Patients with Severe Traumatic Brain Injury in Emergency Hospital Mansoura University Samir Mohamed Attia ${ }^{1}$, Mohamed Farag Selim ${ }^{1}$, Mohamed Elsaied Ibraheim ${ }^{2}$, Mohamed Ibraheim Elsaied Abdulfattah*3 \\ Departments of 1Vascular Surgery, ${ }^{2}$ Critical Care Medicine and ${ }^{3}$ Emergency Medicine, Faculty of Medicine - Mansoura University, Egypt \\ *Corresponding author: Mohamed Ibraheim Elsaied Abdulfattah, Mobile: (+20) 01145014835,
} E-Mail: mohamed.ibraheim100@yahoo.com

\begin{abstract}
Background: Every year more than 10 million people are affected by traumatic brain injuries (TBIs). Despite efforts being made to improve TBI care, it remains a public health problem. Deep venous thrombosis (DVT) is common among trauma patients. If left untreated it may result in lethal pulmonary thromboembolism.

Objective: The aim of the current study was to determine the incidence and risk factors of venous thromboembolism in traumatic brain injury patients.

Patients and methods: This was a prospective study, which was conducted over 200 patients with severe traumatic brain injury admitted to Emergency Hospital Mansoura University over a year from January 2020 to January 2021. Emergency Hospital Mansoura University is a level one trauma center with about 250,000 visit and 25,000 trauma cases per year. Results: RTA and MCA were demonstrated to be the most common modes followed by FFH ad lastly direct trauma. The average Glasgow Coma Scale (GCS) was $6.12 \pm 1.937$ and $69.5 \%$ of the studied cases required intubation. There were no statistically significant differences among both groups (Non-VTE versus VTE) regarding GCS as well as the need for intubation. There were no statistically significant differences among both groups concerning EDH, SDH, ICH and brain contusion, while SAH and brain edema demonstrated significant increase in non-VTE cases compared to VTE cases.
\end{abstract}

Conclusion: The current study demonstrated that, TBI is a life-threatening condition with a high mortality rate and seems to be associated with significant increase in the possibility of DVT development.

Keywords: Incidence and risk factors of venous, Thromboembolism, Traumatic brain injury.

\section{INTRODUCTION}

Traumatic brain injury (TBI) is a leading cause of morbidity and mortality in the trauma population. TBI remains one of the most complex diseases known in the most complex of all organs in the body. TBI is a disruption of brain function and/or structure due to the application of an external physical force that produces signs and symptoms of brain dysfunction in the acute injury period ${ }^{(\mathbf{1})}$. The causes of TBI are many and varied and include penetrating and non-penetrating injuries that are based on their overall level of severity, can evoke different degrees of morbidity, typically framed within the context of the Glasgow coma scale (GCS) score. With the GCS, patients are divided into crude categories of mild, moderate, and severe injury ${ }^{(2)}$.

Although true incidence in the TBI population remains uncertain, there is undoubtedly substantial risk for development of venous thromboembolism (VTE), such as DVT and pulmonary embolism (PE), in those hospitalized after severe TBIs ${ }^{(3)}$. Venous thromboembolism risk after TBI presumably derives from the prevalence of common risk factors in this patient population, including paralysis, immobility, and concurrent orthopedic injuries ${ }^{(4)}$.

Additional relative risk factors are age and blood product transfusions. Severe TBI also exerts a major effect on the physiological dynamics of coagulation and fibrinolysis ${ }^{(5)}$.
The occurrence of VTE extends the length of stay in intensive care units (ICU), general floor beds, and necessitates diagnostic testing and therapeutic interventions ${ }^{(\boldsymbol{6})}$. Adequate prophylaxis is necessary to prevent VTE and subsequent complications because of this increased risk among TBI patients. They are generally divided into mechanical and pharmacological modalities and both are effective. However, while the former is largely without risk, the latter is associated with the risk of fresh or worsening cranial hemorrhage precluding its ubiquitous implementation ${ }^{(7)}$.

The aim of this study was to determine the incidence and risk factors of venous thromboembolism in traumatic brain injury patients.

\section{PATIENTS AND METHODS}

This was a prospective study conducted over 200 patients with severe traumatic brain injury admitted to Emergency Hospital Mansoura University over a year from January 2020 to January 2021. Emergency Hospital Mansoura University is a level one trauma center with about 250,000 visit and 25,000 trauma cases per year.

\section{Ethical approval:}

An approval of the study was obtained from Mansoura University academic and ethical 
committee. Every patient signed an informed written consent for acceptance of the operation.

Inclusion criteria: Patients with severe traumatic brain injury (GCS is less than 9), and patient's age above 18 years.

Exclusion criteria: At least one of the following conditions; those with a diagnosis of primary hypercoagulable state, and persons who came to the emergency hospital dead after severe traumatic brain injury.

Resuscitation of the patient: Airway maintenance \& cervical spine immobilization, breathing and ventilation, circulation and control of hemorrhage, disability: neurological status and Glasgow coma scale (GCS), and exposure / environmental control.

\section{By AMPLE history:}

A = Allergies.

$\mathbf{M}=$ Medication currently used .

$\mathbf{P}=$ Past illnesses $/$ Pregnancy .

$\mathbf{L}=$ Last meal.

$\mathbf{E}=$ Events / Environment related to injury.

Pulmonary embolism: Shortness of breathing (SOB), rapid breathing, cough, coughing up blood, chest pain worsened by breathing.

VTE: Unilateral limb pain, swelling, redness, limited movement.

Clinical Examination and $2^{\text {ry }}$ survey:

Full clinical examination of all body.

\section{In PE:}

- Tachycardia.

- Tachypnea.

- The lungs are usually normal.

- Pleural friction rub may be audible over the affected area of the lung (mostly in PE with infarcts).

- Decreased percussion note, audible breath sound, and vocal resonance maybe detected with pleural effusion.

\section{Venous Thromboembolism Screening:}

Patients admitted to ICU were followed up for two weeks to assess risk factors of VTE. When VTE was suspected by history and complaint, it was confirmed by focused clinical examination and investigation (laboratory and radiological). A standard screening protocol was used to assess for lower

\section{History taking}

extremity DVTs in all patients admitted after a trauma. Specifically, lower extremity venous duplex ultrasound was performed within the first 48 hours of admission, with a second duplex performed during the first week after admission. DVT was defined as an abnormality seen on venous duplex ultrasound such as the presence of dilated, non-compressible veins or intraluminal shadows consistent with thrombosis. Computed tomography angiograms of the chest were used to evaluate for PE as indicated based on clinical suspicion such as the onset of abrupt and unexplained hypoxia, hypotension, tachycardia, or any combination of the aforementioned. Pulmonary embolism was defined as a filling defect detected on a computed tomography pulmonary angiogram.

\section{1}

Radiological investigations: FAST (Focused Assessment with sonography for Trauma), X-ray (chest, pelvis, lumbosacral spine and cervical spine) and multislice CT brain.

\section{Statistical analysis}

IBM's SPSS statistics (Statistical Package for the Social Sciences) for windows (version 25, 2017) was used for statistical analysis of the collected data. Shapiro-Wilk test was used to check the normality of the data distribution. All tests were conducted with 95\% confidence interval. P (probability) value $\leq 0.05$ was considered statistically significant. Charts were generated using SPSS' chart builder and Microsoft Excel for windows 2019. Quantitative variables were expressed as mean and standard deviation, median, inter-quartile range, minimum and maximum as appropriate, while categorical variables were expressed as frequency and percentage. Bivariate correlations were assessed using Pearson's or Spearman's correlation coefficient depending on the nature of data. Odds ratio: Risk assessment was done using odds ratio in the crosstab function. $\mathrm{P}$ value $\leq$ 0.05 was considered significant.

\section{RESULTS}

Table (1) showed the demographic characteristics of the studied sample. The mean age and BMI were $42.02 \pm 13.505$ and $29.82 \pm 5.243$ respectively, with male to female ratio was 75.5/24.5. Thirty percent of cases were smokers.

Table (1): Demographic characteristics of the studied sample

\begin{tabular}{|c|c|c|c|c|c|}
\hline \multicolumn{2}{|c|}{ All patients $(n=200)$} & Mean \pm SD & Median & Range & IQR \\
\hline \multicolumn{2}{|c|}{ Age (years) } & $42.02 \pm 13.505$ & 44.00 & $20.0,64.0$ & $30.00,53.75$ \\
\hline \multicolumn{2}{|l|}{ BMI } & $29.82 \pm 5.243$ & 29.17 & $19.1,47.4$ & $26.22,33.79$ \\
\hline \multirow{2}{*}{ Gender } & Male & \multicolumn{4}{|c|}{$75.5 \%(151)$} \\
\hline & Female & \multicolumn{4}{|c|}{$24.5 \%(49)$} \\
\hline \multicolumn{2}{|c|}{ Smoking } & \multicolumn{4}{|c|}{$15.0 \%(30)$} \\
\hline
\end{tabular}


Table (2) demonstrates the medical history as well as current medication of the studied sample. DM was demonstrated to be the most common medical disease among the studied cases followed by hypercholesterolemia (11.5\%), HTN (10.5\%) and lastly DVT (2.5\%). One percent, $2.5 \%, 7.5 \%$ of the studied cases were receiving platelet, anticoagulants and statins as a treatment respectively.

Table (2): Medical history and current medication of the studied sample

\begin{tabular}{|l|c|c|}
\hline \multicolumn{1}{|c|}{ All patients $(\mathbf{n = 2 0 0})$} & Frequency & Percentage \\
\hline DM & 29 & $14.5 \%$ \\
\hline HTN & 21 & $10.5 \%$ \\
\hline Hypercholesterolemia & 23 & $11.5 \%$ \\
\hline DVT & 5 & $2.5 \%$ \\
\hline Anti-Platelet & 2 & $1.0 \%$ \\
\hline Anticoagulants & 6 & $3.0 \%$ \\
\hline Statins & 15 & $7.5 \%$ \\
\hline Data were expressed as percentage and frequency.
\end{tabular}

Concerning mode of trauma of the studied sample, table (3) revealed that road traffic accidents (RTAs) (34.5\%) and motor cycle accident (MCA) (34.5\%) were demonstrated to be the most common modes followed by fall from height $(\mathrm{FFH})(17.5 \%)$ and lastly direct trauma $(13.5 \%)$.

Table (3): Mode of trauma of the studied sample

\begin{tabular}{|l|c|c|}
\hline \multicolumn{1}{|c|}{ All patients $(\mathbf{n = 2 0 0})$} & Frequency & Percentage \\
\hline FFH & 35 & $17.5 \%$ \\
\hline RTA & 69 & $34.5 \%$ \\
\hline MCA & 69 & $34.5 \%$ \\
\hline Direct trauma & 27 & $13.5 \%$ \\
\hline Data were expressed as percentage and frequency.
\end{tabular}

Table (4) demonstrated that the mean GCS was $6.12 \pm 1.937$ and $69.5 \%$ of the studied cases required intubation.

Table (4): GCS and need for intubation among the studied sample

\begin{tabular}{|l|c|c|c|}
\hline \multicolumn{1}{|c|}{ All patients $(\mathbf{n}=\mathbf{2 0 0})$} & Mean \pm SD & Median & IQR \\
\hline GCS & $6.12 \pm 1.937$ & 6.00 & $4.00,8.00$ \\
\hline Need for intubation & \multicolumn{3}{l}{$69.5 \%(139)$} \\
\hline $\begin{array}{l}\text { Data were expressed as mean and standard deviation, median, range and interquartile range or as } \\
\text { percentage and frequency. }\end{array}$
\end{tabular}

Regarding results of CT brain in the studied sample, table (5) demonstrated that subdural hematoma (SDH) and brain contusion were demonstrated to be the most frequent CT brain finding, followed by epidural hematoma $(\mathrm{EDH})(24 \%)$, then subarachnoid hematoma (SAH) (17.5) and lastly intracerebral hematoma (ICH) (17\%).

Table (5): Results of CT brain in the studied sample

\begin{tabular}{|l|c|c|}
\hline \multicolumn{1}{|c|}{ All patients $(\mathbf{n = 2 0 0})$} & Frequency & Percentage \\
\hline EDH & 48 & $24.0 \%$ \\
\hline SDH & 72 & $36.0 \%$ \\
\hline SAH & 35 & $17.5 \%$ \\
\hline ICH & 34 & $17.0 \%$ \\
\hline Brain Contusion & 72 & $36.0 \%$ \\
\hline Brain Edema & 141 & $70.5 \%$ \\
\hline Data were expressed as percentage and frequency.
\end{tabular}

Table (6) illustrated associated trauma in the studied sample. Most of the studied cases had no associated trauma (41.5\%), while fracture, pneumothorax, hemothorax and free fluid were reported to be present in (34\%), $(5 \%),(2 \%)$ and $(17.5 \%)$ of cases respectively. 
Table (6): Associated trauma in the studied sample

\begin{tabular}{|l|c|c|}
\hline \multicolumn{1}{|c|}{ All patients $(\mathbf{n = 2 0 0 )}$} & Frequency & Percentage \\
\hline None & 83 & $41.5 \%$ \\
\hline Fracture & 68 & $34.0 \%$ \\
\hline Pneumothorax & 10 & $5.0 \%$ \\
\hline Hemothorax & 4 & $2.0 \%$ \\
\hline Free fluid & 35 & $17.5 \%$ \\
\hline Data were expressed as percentage and frequency.
\end{tabular}

Table (7) illustrated the initial clinical and laboratory assessment in the studied sample. The mean SBP, Hb, WBC, PLT, INR and Creatinine were $124.80 \pm 9.076,11.20 \pm 1.434,11.03 \pm 1.855,262.31 \pm 49.127,1.29 \pm 0.174$ and $1.32 \pm 0.290$ respectively.

Table (7): Initial clinical and laboratory assessment in the studied sample

\begin{tabular}{|l|c|c|c|}
\hline \multicolumn{1}{|c|}{ All patients $(\mathbf{n = 2 0 0})$} & Mean $\mathbf{\pm}$ SD & Median & IQR \\
\hline SBP $($ cells/ $\boldsymbol{\mu L})$ & $124.80 \pm 9.076$ & 125.00 & $120.00,130.00$ \\
\hline Hb $(\mathbf{g} / \mathbf{d L})$ & $11.20 \pm 1.434$ & 11.10 & $10.03,12.48$ \\
\hline WBC $(\mathbf{m c L})$ & $11.03 \pm 1.855$ & 11.00 & $9.60,12.68$ \\
\hline PLT $(\mathbf{m c L})$ & $262.31 \pm 49.127$ & 264.00 & $1.14,1.43$ \\
\hline INR & $1.29 \pm 0.174$ & 1.30 & $1.12,1.49$ \\
\hline Creatinine $(\boldsymbol{\mu m o l} / \mathbf{k g} /$ day) & $1.32 \pm 0.290$ & 1.31 & \\
\hline \multicolumn{4}{|l}{} \\
Data were expressed as mean and standard deviation, median, range and interquartile range.
\end{tabular}

Table (8) showed the management in the studied sample where the majority of which were managed conservatively ( $70.5 \%)$, while only $29.5 \%$ were operatively managed.

Table (8): Management in the studied sample

\begin{tabular}{|l|c|c|}
\hline \multicolumn{1}{|c|}{ All patients $(\mathbf{n = 2 0 0})$} & Frequency & Percentage \\
\hline Conservative & 141 & $70.5 \%$ \\
\hline Operative & 59 & $29.5 \%$ \\
\hline Data were expressed as percentage and frequency.
\end{tabular}

Table (9) displayed the results of duplex US and CT pulmonary angiography in the studied sample. Regarding, 1st Duplex 48h after admission, entire cases were normal. While second duplex one week after admission, 79\% were normal, 6\% had femoral vein DVT, 6\% had popliteal vein DVT and 9\% had GSV vein DVT. Concerning CT pulmonary angiography, $97 \%$ of cases were normal, while, $3 \%$ had thromboembolism.

Table (9): Results of duplex US and CT pulmonary angiography in the studied sample

\section{All patients $(n=200)$}

\begin{tabular}{|c|c|c|c|}
\hline \multicolumn{2}{|c|}{ All patients $(n=200)$} & Frequency & Percentage \\
\hline $1^{\text {st }}$ Duplex $48 \mathrm{~h}$ after admission & Normal & 200 & $100.0 \%$ \\
\hline \multirow{4}{*}{$\begin{array}{l}\text { Second duplex one } \\
\text { week after admission }\end{array}$} & Normal & 158 & $79.0 \%$ \\
\hline & Femoral vein DVT & 12 & $6.0 \%$ \\
\hline & Popliteal vein DVT & 12 & $6.0 \%$ \\
\hline & GSV vein DVT & 18 & $9.0 \%$ \\
\hline \multirow{2}{*}{ CT pulmonary angiography } & Normal & 194 & $97.0 \%$ \\
\hline & Thromboembolism & 6 & $3.0 \%$ \\
\hline
\end{tabular}

Table (10) illustrated the length of hospital and ICU stay and final outcome in the studied sample. The mean length of hospital stay period, and intensive care unit length of stay in terms of outcome were $16.69 \pm 3.905$ and $14.11 \pm 4.357$ respectively. The majority of cases survived (65\%), while only $35 \%$ died.

Table (10): Length of hospital and ICU stay and final outcome in the studied sample

\begin{tabular}{|c|c|c|c|c|}
\hline All & 200) & Mean \pm SD & Median & IQR \\
\hline Hospital & y (days) & $16.69 \pm 3.905$ & 17.00 & $14.00,19.00$ \\
\hline Intensive care & of stay (days) & $14.11 \pm 4.357$ & 14.00 & $11.00,17.00$ \\
\hline \multirow{2}{*}{ Outcome } & Survival & \multicolumn{3}{|c|}{$65.0 \%(130)$} \\
\hline & Death & \multicolumn{3}{|c|}{$35.0 \%(70)$} \\
\hline
\end{tabular}

Data were expressed as mean and standard deviation, median, range and interquartile range or as percentage and frequency.

Table (11) illustrated the correlation between occurrence of thromboembolism in CT pulmonary angiography and quantitative variables. There were no statistically significant correlations among occurrence of thromboembolism in CT pulmonary angiography and all studied variables $(\mathrm{P}>0.05)$. 
Table (11): Correlation between occurrence of thromboembolism in CT pulmonary angiography and quantitative variables

\begin{tabular}{|c|c|c|}
\hline All patients $(n=200)$ & Correlation coefficient & $\mathbf{P}$ \\
\hline Age (years) & -0.039 & 0.580 \\
\hline BMI & 0.048 & 0.498 \\
\hline GCS & 0.035 & 0.622 \\
\hline SBP $($ cells $/ \mu L)$ & 0.004 & 0.956 \\
\hline Hb (g/dL) & -0.022 & 0.757 \\
\hline WBC (mcL) & 0.086 & 0.227 \\
\hline PLT (mcL) & 0.025 & 0.729 \\
\hline INR & -0.078 & 0.272 \\
\hline Creatinine $(\mu \mathrm{mol} / \mathrm{kg} / \mathrm{day})$ & -0.027 & 0.705 \\
\hline Hospital length of stay (days) & -0.069 & 0.333 \\
\hline Intensive care unit length of stay (days) & 0.023 & 0.752 \\
\hline
\end{tabular}

Table (12) illustrated the odds ratio of occurrence of thromboembolism in CT pulmonary angiography according to qualitative variables. It was demonstrated that MCA, need for intubation, EDH, brain edema, fracture, hemothorax, femoral vein DVT, popliteal vein DVT, GSV vein DVT were positively correlated with occurrence of thromboembolism in CT pulmonary angiography. On the contrary, RTA and no associated trauma were negatively correlated with occurrence of thromboembolism in CT pulmonary angiography.

Table (12): Odds ratio of occurrence of thromboembolism in CT pulmonary angiography according to qualitative variables

\begin{tabular}{|l|c|}
\hline \multicolumn{1}{|c|}{ All patients $(\mathbf{n}=\mathbf{2 0 0})$} & Odds ration \\
\hline Female gender & 0.960 \\
\hline Smoking & 1.138 \\
\hline DM & 1.186 \\
\hline HTN & 0.966 \\
\hline Hypercholesterolemia & 0.966 \\
\hline DVT & 0.969 \\
\hline Platelet & 0.970 \\
\hline Anticoagulants & 0.969 \\
\hline Statins & 0.968 \\
\hline FFH & 0.964 \\
\hline RTA & $\mathbf{0 . 3 7 1}$ \\
\hline MCA & $\mathbf{3 . 9 6 9}$ \\
\hline Direct trauma & 1.292 \\
\hline Need for intubation & $\mathbf{2 . 2 7 9}$ \\
\hline EDH & $\mathbf{3 . 3 1 1}$ \\
\hline SDH & 0.886 \\
\hline SAH & 0.964 \\
\hline ICH & 0.976 \\
\hline Brain Contusion & 0.886 \\
\hline Brain Edema & $\mathbf{2 . 3 9 0}$ \\
\hline No Associated trauma & $\mathbf{0 . 2 7 3}$ \\
\hline Fracture & $\mathbf{1 . 9 8 5}$ \\
\hline Pneumothorax & 0.968 \\
\hline Hemothorax & $\mathbf{1 2 . 7 3 3}$ \\
\hline Free fluid & 0.941 \\
\hline Surgical Management & 5.055 \\
\hline Normal Duplex & 0.857 \\
\hline Femoral vein DVT & $\mathbf{3 . 3 2 7}$ \\
\hline Popliteal vein DVT & $\mathbf{2 0 . 5 5 6}$ \\
\hline GSV vein DVT & $\mathbf{5 . 5 6 3}$ \\
\hline
\end{tabular}




\section{DISCUSSION}

Regarding the demographic characteristics of the studied sample, the mean age and BMI were $42.02 \pm 13.505$ years and $29.82 \pm 5.243 \mathrm{~kg} / \mathrm{m}^{2}$ respectively; with male to female ratio was $75.5 / 24.5 \%$. Thirty percent of cases were smokers. In addition, there were no statistically significant differences among both groups (Non-VTE versus VTE) regarding age, BMI, gender and smoking. Such fact indicated that both groups were comparable and demographic features were not interfering with the net result of the study.

In terms of the medical history as well as current medication of the studied sample, DM was demonstrated to be the most common medical disease among the studied cases followed by hypercholesterolemia $(11.5 \%)$ and HTN $(10.5 \%)$ and lastly DVT (2.5\%). One percent, $2.5 \%, 7.5 \%$ of the studied cases were receiving platelet, anticoagulants and statins as a treatment respectively. In addition, both groups demonstrated insignificant differences in terms of all medical history $(\mathrm{P}>0.05)$ with the exclusion of previous DVT being highly increased in VTE group (P $=0.007)$. Moreover, both groups demonstrated insignificant differences in terms of all current medications $(P>0.05)$. Such result indicated that both groups were comparable and medical history was not interfering with the net result of the study. Of note the percentage of previous DVT among the studied cases was $2.5 \%$, the majority of which developed VTE compared to only one case in non-VTE group.

It was reported that, DVT is common in persons with TBI, with an incidence as high as $54 \%$. In patients with TBI, risk factors for DVT include immobility, lower extremity fracture, paralysis, and disruption in coagulation and fibrinolysis ${ }^{(8)}$. In the same line, Reiff et $\boldsymbol{a l} .{ }^{(9)}$ demonstrated that, irrespective of the time of initiation of pharmacologic prophylaxis, TBI is independently associated with the formation of DVT. A threefold to fourfold increased risk of DVT formation is consistent across all prophylaxis groups among patients with TBI. Denson et al. ${ }^{(\mathbf{1 0 )})}$ reported a DVT incidence of $25 \%$ among patients with isolated TBI, with $42 \%$ of their population receiving pharmacologic prophylaxis after a mean time of 14 days. The alteration among results may be due to the fact that, a trend of increasing DVT risk with increasing time to initiation of prophylaxis. In patients with TBI, the absolute risk is increased from $3.6 \%$ in " 0 hours to $4.5 \%$ in " 24 hours to $15.4 \%$ in 48 hours. Delayed institution of prophylaxis in trauma patients has been suggested to contribute to the development of symptomatic VTE and fatal PE ${ }^{(\boldsymbol{9}, 11)}$. While, Ekeh et al. ${ }^{(12)}$ demonstrated higher incidence of DVT among TBI cases and demonstrated that the incidence of DVT was present in $31.6 \%$ of their cases (one third of cases). Such increase in incidence in Ekeh et al. ${ }^{(12)}$ research compared to the current study may be due to the fact that they conducted their study on cases with moderate to severe head trauma, while the current study was performed on all TBI cases.

Concerning mode of trauma of the studied sample, the current study revealed that RTA was $34.5 \%$ and MCA was $34.5 \%$ followed by FFH $(17.5 \%)$ ad lastly direct trauma (13.5\%). In addition, both groups demonstrated insignificant differences regarding mode of trauma $(P>0.05)$. It has been shown that in lowmiddle income countries in the past 17 years, violence and road traffic accidents (RTAs) have been the main causes of TBI and have associated high mortality ${ }^{(13)}$. Similarly, Dunne et al. ${ }^{(14)}$ revealed that, RTA is a common cause of TBI in Latin America. They demonstrated that, TBI due to RTAs is more frequent in males between the ages of 15 and 35 years, and patients in motor vehicles accounted for most cases, followed by pedestrians, motorcyclists, and cyclists. On the contrary, El Mestoui et al. ${ }^{(15)}$ demonstrated that, the most common mechanism of injury involved fall from height, followed by bicycle accidents.

There is a clear regional variation in the disease impact; not only low- and middle-income areas do have a higher incidence of TBI but also are associated with greater morbimortality. A reason for this may be the lower level of acute health care provision in these areas, lack of access to existing health care systems, and disjointed or nonexistent preventative measures ${ }^{(\mathbf{1 5})}$.

The current study demonstrated that the mean GCS was $6.12 \pm 1.937$ and $69.5 \%$ of the studied cases required intubation. In addition, there were no statistically significant differences among both groups (Non-VTE versus VTE) regarding GCS as well as the need for intubation $(\mathrm{P}>0.05)$.

Early recognition of TBI significantly affects clinical outcome. Accurate assessment of the probability of TBI at triage permits more efficient resource utilization for patients with head trauma. Traditionally, GCS has been used to rapidly identify patients with TBI at triage ${ }^{(16)}$.

Regarding results of CT brain in the studied sample, the current study demonstrated that SDH and brain contusion were demonstrated to be the most frequent CT brain finding, followed by EDH (24\%), then SAH (17.5\%) and lastly ICH (17\%). In addition, there were no statistically significant differences among both groups concerning EDH, SDH, ICH and brain contusion $(\mathrm{P}>0.05)$, while SAH and brain edema demonstrated significant increase in non-VTE cases compared to VTE cases $(\mathrm{P}<0.05)$. The current study come in accordance with a recent research, which was conducted by Adatia et al. ${ }^{(17)}$ and demonstrated that, progression of cerebral contusions occurs in up to $75 \%$ of patients with TBI whatever the age and this contributes to subsequent clinical deterioration and requirement for surgical intervention. In the same line, Ohaegbulam et al. ${ }^{(18)}$ demonstrated in their study on 
TBI cases that the most common CT findings were subdural hematoma $30 \%$, cerebral contusions and edema $30.7 \%$, skull fractures $23.4 \%$ and extradural hematoma $8.0 \%$. About $64 \%$ of the CT findings required surgical interventions. The overall mortality was $11.1 \%$, but amongst the 137 patients who had abnormal CT findings, it was $13.9 \%$. While, Gupta et al. ${ }^{(19)}$ demonstrated in their study on TBI cases that, CT scans revealed skull fractures $(62.04 \%)$, intracerebral hematoma (46.33\%), epidural hematoma (30.36\%), subdural hematoma (19.37\%), subarachnoid hematoma (28.79\%), diffuse axonal injury, brain swelling and edema (63.35\%), midline shift (24.34\%), pneumocranium $(12.04 \%)$ and intra-ventricular hemorrhage (10.73\%).

In terms of associated trauma in the studied sample, most of the studied cases had no associated trauma (41.5\%), while fracture, pneumothorax, hemothorax and free fluid were reported to be present in $(34 \%),(5 \%),(2 \%)$ and $(17.5 \%)$ of cases respectively. In addition, VTE cases demonstrated highly statistically significant increases in associated trauma compared to non-VTE ones (82 versus 52.5) (P $<0.001)$. Of note, Patients with TBI with polytrauma are at greater risk for 3- and 6-month disability compared to those with isolated TBI ${ }^{(20)}$. In the same line, Groswasser et al. $^{(\mathbf{2 1 )}}$ conducted their study on 328 patients with severe TBI. They reported that, 58\% had associated trauma, mostly in the skeletal system. The presence of one single associated trauma had no additional effect on rehabilitation as evaluated by actual work placement. In contrast, multiple lesions were liked with a less favorable outcome, probably due to a greater severity of the initial CNS damage.

As regards, the management in the studied sample in which the majority were managed conservatively $(70.5 \%$ ), while only $29.5 \%$ were operatively managed. In addition, there were statistically significant differences among both groups regarding the method of management as the conservative management increased in non-VTE cases, while operative ones increased in VTE cases.

Regarding the results of duplex US and CT pulmonary angiography in the studied sample, the 1st Duplex $48 \mathrm{~h}$ after admission, entire cases were normal, while, second duplex one week after admission 79\% were normal, $6 \%$ had femoral vein DVT, $6 \%$ had popliteal vein DVT and 9\% had GSV vein DVT. Concerning CT pulmonary angiography, $97 \%$ of cases were normal, while, $3 \%$ had thromboembolism. In addition, there were no statistically significant correlations among occurrence of thromboembolism in CT pulmonary angiography and all studied variables $(\mathrm{P}$ $>0.05)$.

Contrast venography is the gold standard for the diagnosis of DVT. This modality is infrequently used due to its complications, patient discomfort, and cost. Duplex US is $89 \%$ to $96 \%$ sensitive, and $96 \%$ to $100 \%$ specific in the diagnosis of DVT of significant size to produce symptomatic DVT and is the most frequently used diagnostic test ${ }^{(22)}$. In the same line, Ekeh et al. ${ }^{(12)}$ demonstrated that, the majority of DVTs (84.2\%) occurred in the lower extremity, with the majority of these occurring below the knee (54\% soleal $26.9 \%$, peroneal $17.5 \%$, posterior tibial $8.0 \%$, and gastrocnemius $0.005 \%$ ). Femoral DVTs were present in $24 \%$ of patients. Upper extremity DVTs occurred in $15.8 \%$ of patients

Regarding the length of hospital and ICU stay and final outcome in the studied sample, the mean hospital length of stay period, and intensive care unit length of stay in terms of outcome were $16.69 \pm 3.905$ and $14.11 \pm 4.357$ respectively. The majority of cases survived (65\%), while only $35 \%$ died. While, Levant et al. ${ }^{(23)}$ reported that, the average length of stay for inpatients with a first-listed diagnosis of TBI with a stay in the ICU was 7.3 days, with an average of 1.2 days in the ICU. The average length of stay for inpatients hospitalized for TBI without a stay in the ICU was 3.2 days.

With regard to the odds ratio of occurrence of thromboembolism in CT pulmonary angiography according to qualitative variables, it was demonstrated that MCA, need for intubation, EDH, brain edema, fracture, hemothorax, femoral vein DVT, popliteal vein DVT, GSV vein DVT were positively correlated with occurrence thromboembolism in CT pulmonary angiography. On the contrary, RTA and no associated trauma were negatively correlated with occurrence of thromboembolism in CT pulmonary angiography. While, Ekeh et al. ${ }^{(12)}$ demonstrated that, older age, male gender, higher ISS, and the presence of a lower extremity injury are strong predictors for developing DVT.

\section{CONCLUSION}

The current study demonstrated that TBI is a life-threatening condition with a high mortality rate and seems to be associated with significant increase in the possibility of DVT development. In addition, MCA, need for intubation, EDH, brain edema, fracture, hemothorax, femoral vein DVT, popliteal vein DVT, GSV vein DVT were positively correlated with occurrence thromboembolism.

\section{REFERENCES}

1. McGinn M, Povlishock J (2016): Pathophysiology of traumatic brain injury. Neurosurgery Clinics, 27 (4): 397 407.

2. Rosenfeld J, Maas A, Bragge $P$ et al. (2012): Early management of severe traumatic brain injury. The Lancet, 380 (9847): 1088-1098.

3. Eapen C, Hong S, Jaramillo C et al. (2018): Medical Complications After Moderate to Severe Traumatic Brain Injury. Rehabilitation After Traumatic Brain Injury, Pp: 
$23-36$

https://www.researchgate.net/publication/330042801_M edical_Complications_After_Moderate_to_Severe_Trau matic_Brain_Injury

4. Phelan H (2012): Pharmacologic venous thromboembolism prophylaxis after traumatic brain injury: a critical literature review. Journal of Neurotrauma, 29 (10): 1821-1828.

5. Maegele $M$ (2013): Coagulopathy after traumatic brain injury: incidence, pathogenesis, and treatment options. Transfusion, 53: 28-37.

6. Prabhu V, Loftus M (2016): Venous Thromboembolism Prophylaxis in Neurosurgery. In Anticoagulation and Hemostasis in Neurosurgery : Springer, Cham. Pp: 327-342. https://link.springer.com/book/10.1007/978-3-31927327-3\#editorsandaffiliations

7. Toker S, Hak D, Morgan S (2011): Deep vein thrombosis prophylaxis in trauma patients. Thrombosis, 11: 505373-8.

8. Cifu D, Kaelin D, Wall B (1996): Deep venous thrombosis: incidence on admission to a brain injury rehabilitation program. Archives of Physical Medicine and Rehabilitation, 77 (11): 1182-1185.

9. Reiff D, Haricharan R, Bullington $N$ et al. (2009): Traumatic brain injury is associated with the development of deep vein thrombosis independent of pharmacological prophylaxis. Journal of Trauma and Acute Care Surgery, 66 (5): 1436-1440.

10. Denson K, Morgan D, Cunningham R et al. (2007): Incidence of venous thromboembolism in patients with traumatic brain injury. The American Journal of Surgery, 193 (3): 380-384.

11. Yablon S, Rock W, Nick T et al. (2004): Deep vein thrombosis: Prevalence and risk factors in rehabilitation admissions with brain injury. Neurology, 63 (3): 485491.

12. Ekeh A, Dominguez K, Markert $\mathrm{R}$ et al. (2010): Incidence and risk factors for deep venous thrombosis after moderate and severe brain injury. Journal of Trauma and Acute Care Surgery, 68 (4): 912-915.
13. Hyder A, Wunderlich $C$, Puvanachandra $P$ et al. (2007): The impact of traumatic brain injuries: a global perspective. Neuro Rehabilitation, 22 (5): 341-353.

14. Dunne J, Quiñones-Ossa G, Still E et al. (2020): The epidemiology of traumatic brain injury due to traffic accidents in Latin America: a narrative review. Journal of Neurosciences in Rural Practice, 11 (2): 287-94.

15. El Mestoui Z, Jalalzadeh H, Giannakopoulos G et al. (2017): Incidence and etiology of mortality in polytrauma patients in a Dutch level I trauma center. European Journal of Emergency Medicine, 24 (1): 49-54.

16. Zehtabchi S, Sinert R, Soghoian S et al. (2007): Identifying traumatic brain injury in patients with isolated head trauma: are arterial lactate and base deficit as helpful as in polytrauma?. Emergency Medicine Journal, 24(5): 333-335.

17. Adatia K, Newcombe V, Menon D (2020): Contusion progression following traumatic brain injury: a review of clinical and radiological predictors, and influence on outcome. Neurocritical Care, 34: 5-9.

18. Ohaegbulam S, Mezue W, Ndubuisi C et al. (2011): Cranial computed tomography scan findings in head trauma patients in Enugu, Nigeria. Surgical Neurology International, 2: 182-188.

19. Gupta P, Krishna A, Dwivedi A et al. (2011): CT scan findings and outcomes of head injury patients: A cross sectional study. Journal of Pioneering Medical Sciences, 1 (3): 78-85.

20. Yue J, Satris G, Dalle O et al. (2020): Polytrauma is associated with increased three-and six-month disability after traumatic brain injury: a TRACK-TBI pilot study. Neurotrauma Reports, 1 (1): 32-41.

21. Groswasser Z, Cohen M, Blankstein E (1990): Polytrauma associated with traumatic brain injury: incidence, nature and impact on rehabilitation outcome. Brain Injury, 4 (2): 161-166.

22. Sharma O, Oswanski M, Joseph R et al. (2007): Venous thromboembolism in trauma patients. The American Surgeon, 73 (11): 1173-1180.

23. Levant S, Chari K, DeFrances C (2016): National Hospital Care Survey Demonstration Projects: Traumatic Brain Injury. National Health Statistics Reports, 97: 1-16. 